\title{
Elevated PIVKA-II is Associated with Early Recurrence and Poor Prognosis in BCLC 0-A Hepatocellular Carcinomas
}

\author{
Bei-Li Wang, Qi-Wen Tan, Xing-Hui Gao, Jiong Wu, Wei Guo*
}

\begin{abstract}
Background: To investigate the prognostic value of serum PIVKA-II (prothrombin induced by the absence of vitamin K or antagonist-II) in BCLC (Barcelona Clinic Liver Cancer) 0-A hepatocellular carcinoma (HCC) patients after curative resection. Materials and Methods: Preoperative sera were collected from 140 patients with BCLC 0-A HCCs undergoing curative resection during 2011-2012 in Zhongshan Hospital. Follow-up ended on November 2013. ELISA was used to detect the serum concentrations of preoperative PIVKA-II. The prognostic value of PIVKA-II and other clinicopathological factors was analyzed by the Kaplan-Meier method and the multivariate Cox proportional hazards model. Results: During follow-up, 39 of 140 patients suffered recurrence and the 1-year recurrence rate was $27.9 \%$. The high-PIVKA-II expression group had lower 1-year time to progression (TTP) compared with the low-expression group $(\mathbf{5 4 . 8 \%}$ vs $20.2 \%, p<0.001)$. Patients with high preoperative PIVKA-II expression showed a relatively higher risk of developing postoperative recurrence than those with low expression in the low-recurrence-risk subgroups, including $\alpha$-fetoprotein $\leq 400 \mathrm{ng} / \mathrm{mL}(\mathbf{4 5 . 4 \%} v \mathrm{~s}$ $16.7 \%$; $\boldsymbol{p}=\mathbf{0 . 0 0 6})$, tumor size $\leq 5 \mathrm{~cm}(\mathbf{5 4 . 2 \%}$ vs $18.1 \%$; $\boldsymbol{p}<0.001)$, single tumor $(\mathbf{5 6 . 0 \%}$ vs $\mathbf{1 9 . 1 \% ;} \boldsymbol{p}<0.001)$, absence of satellite lesions $(53.3 \%$ vs $19.8 \%$; $\boldsymbol{p}=\mathbf{0 . 0 0 1})$, absence of vascular invasion $(52.6 \%$ vs $14.9 \%$; $\boldsymbol{p}=\mathbf{0 . 0 0 2})$, and Edmondson stage I/II (60.9\% vs $20.3 \%$; $\boldsymbol{p}<0.001)$. PIVKA-II was the strongest independent prognostic factor for TTP (hazard ratio, $2.877 ; 95 \%$ CI 1.524-5.429; $p=0.001$ ). Conclusions: Elevated PIVKA-II is associated with early recurrence of BCLC 0-A HCC after curative resection and can be considered a novel prognostic predictor.
\end{abstract}

Keywords: Hepatocellular carcinoma - PIVKA-II - BCLC; prognosis - recurrence

Asian Pac J Cancer Prev, 15 (16), 6673-6678

\section{Introduction}

Hepatocellular carcinoma (HCC) is the fifth most common cancer worldwide, with an increasing incidence in recent years and dismal outcomes (Siegel et al., 2012). Surgery remains the most effective treatment with curative potential, but only $10-20 \%$ of HCC patients are eligible for surgical intervention owing to the lack of effective early diagnosis. For the remaining patients, transcatheter arterial chemoembolization (TACE), radiotherapy (RT), or sorafenib are common treatment choices (Bruix et al., 2011). Despite improvements in surveillance and clinical treatment strategies, the prognosis of HCC remains poor because of the high incidence of recurrence and metastasis. Traditional clinicopathological parameters such as tumor morphology, histopathological features, radiological modalities, and tumor staging system offer limited information for predicting postoperative recurrence (Cha et al., 2003). Therefore, it is imperative to develop new approaches for discriminating high-risk factors in patients with recurrence.

The Barcelona Clinic Liver Cancer (BCLC) staging system has become widely accepted in clinical practice and is recommended for prognostic prediction and treatment allocation. It is also used in many clinical trials of new drugs for HCC. The BCLC staging system can be applied to most HCC patients, as long as consideration is given to special subpopulations (e.g., liver transplantation). Patients at very early Stage 0 and early Stage A are optimal candidates for a radical approach. Radical therapy can change the course of HCC, leading to favorable longterm outcomes (Bruix et al., 2011). Patients with BCLC 0 -A cancer are generally thought to have good prognosis after surgical resection, but many studies have reported variable results (Takayama et al., 2008; Santi et al., 2010). Potentially important prognostic factors such as tumor size, multifocal tumors, and vascular invasion may play a major role in these patients.

Tumor markers are used in the diagnosis and staging of cancer, in monitoring therapeutic effectiveness, in detecting recurrence, and in predicting prognosis. $\alpha$-Fetoprotein (AFP) was first introduced as a serological marker for HCC and has since been used in clinical practice both for diagnosis and as an indicator of tumor response to treatment (Gupta et al., 2013; Shi et al., 2014; Wang et al., 2014). Previous studies have demonstrated that PIVKA-II (prothrombin induced by the absence of vitamin $\mathrm{K}$ or antagonist-II) is also an effective marker 
for HCC, and that an elevated PIVKA-II level appears to predict worse tumor behavior.

Additionally, recent molecular studies of PIVKAII have revealed the usefulness of this molecule as a diagnostic marker, as well as its significant role in cancer progression (Wang et al., 2009a; Bertino et al., 2012; Matsubara et al., 2012). However, it remains to be confirmed whether the prognostic predictive value of the PIVKA-II level can be generalized to patients with BCLC 0-A HCC undergoing surgery.

The purpose of the present study was to explore the prognostic significance of PIVKA-II level in patients with BCLC 0-A HCC undergoing surgery, and to analyze the value of PIVKA-II level for predicting the risk of early recurrence after hepatectomy.

\section{Materials and Methods}

\section{Study design}

From March 2011 to October 2012, 261 HCC patients were recruited into this prospective study and underwent curative resection. Curative resection was defined as removal of all recognizable tumors. HCC was diagnosed on the basis of tumor markers and a combination of typical imaging findings on ultrasonography, and dynamic contrast-enhanced computed tomography (CT), according to the American Association for the Study of Liver Diseases (AASLD) guidelines. Disease was stratified according to the 2011 BCLC staging classification. We only focused on patients with BCLC 0-A HCC. Patients with pathologically proven BCLC B-D cancer, or patients who were lost to follow-up after hepatectomy were excluded. The demographic, preoperative laboratory, and pathology data of all patients were collected from electronic medical records. Liver function was evaluated by the Child-Pugh classification system. Of the 261 patients, 140 were finally entered into the analyses, and 121 were excluded for the following reasons: 75 patients were classified as BCLC B-D, and 46 were lost to followup after discharge.

For evaluation of prognostic value, follow-up was completed in November 2013. Time to recurrence (TTR) was defined as the interval between surgery and the diagnosis of any type of recurrence, with intrahepatic recurrence and extrahepatic metastasis defined as the
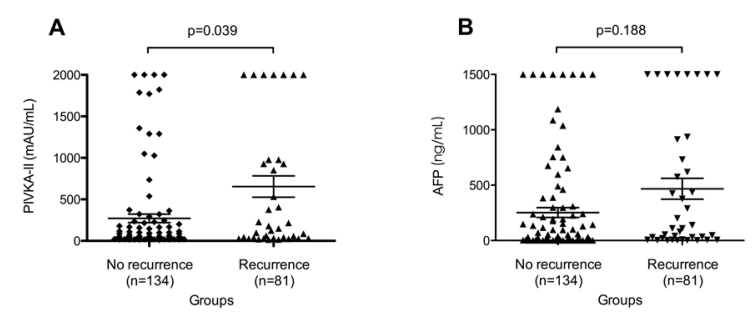

Figure 1. PIVKA-II Levels Correlated Significantly with Early Recurrence. A) Distribution of PIVKAII levels in early recurrent patients $(\diamond)$, nonrecurrent patients $(\mathbf{\Delta})(p=0.039)$. B) Distribution of AFP levels in early recurrent patients $(\boldsymbol{\Delta})$, nonrecurrent patients $(\boldsymbol{\nabla})$ $(p=0.188)$ end points for TTR. Progression-free survival (PFS) was defined as the time between the date of receiving treatment and the date of clinical disease progression, or the date of the last follow-up visit if progression did not occur during follow-up. Approval for the use of human subjects was obtained from the Research Ethics Committee of Zhongshan Hospital, and informed consent was obtained from each individual enrolled in this study.

\section{PIVKA-II determination}

The serum concentrations of PIVKA-II were determined by enzyme immunoassay (Eisai Co., Tokyo, Japan; cutoff value $40 \mathrm{mAU} / \mathrm{mL}$ ) according to the manufacturer's instructions. The level of PIVKA-II was measured when patients were initially diagnosed with HCC, and regular follow-up measurements after treatment were done in some patients.

\section{Statistical analysis}

Statistical analysis was performed using SPSS version 20.0 (IBM, Chicago, IL, USA). Experimental values are presented as mean \pm SEM. $\chi^{2}$ tests and Fisher's exact probability test were used for comparison between groups, as appropriate. The relationship between TTR or PFS and the level of PIVKA-II was analyzed by Kaplan-Meier survival curves and the log-rank test. Results are expressed as hazard ratios (HRs) from the Cox models, along with 95\% CI. Receiver operating characteristic (ROC) curve analysis was used to determine the predictive value of the parameters, and the differences in the area under the curve (AUC) were detected. Two-sided $p$ values $<0.05$ were considered statistically significant.

\section{Results}

Patient demographics and tumor characteristics

Patient demographics are listed in (Table 1). The main cause of $\mathrm{HCC}$ was chronic infection with hepatitis B virus $(\mathrm{n}=127,90.7 \%)$. Most patients were Child-Pugh class A and only seven patients were class B. Fourteen patients were classified as BCLC 0 , and the remaining 126 were BCLC A. By the time of analysis, early recurrence had occurred in 39 of 140 patients, with a mean follow-up time

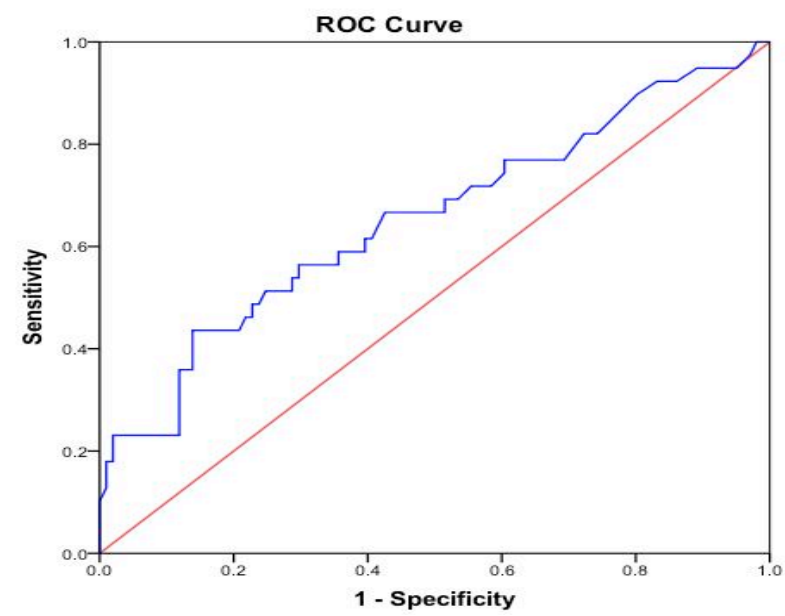

Figure 2. ROC Curves of PIVKA-II for Diagnosis of HCC Early Recurrence. AUC of PIVKA-II was 0.653 
Elevated PIVKA-II is Associated with Early Recurrence and Poor Prognosis in BCLC 0-A Hepatocellular Carcinomas

Table 1. Clinical Characteristics of BCLC Stage 0-A HCC Patients and Correlation with PIVKA-II Levels

\begin{tabular}{|c|c|c|c|c|}
\hline Variable & $\begin{array}{l}\text { No. of } \\
\text { patients } \\
-140\end{array}$ & $\begin{array}{c}\text { PIVKA-II } \\
<373.5 \mathrm{mAU} / \mathrm{mL} \geq \\
(\mathrm{n}=109)\end{array}$ & $\begin{array}{c}\text { PIVKA-II } \\
373.5 \mathrm{mAU} / \mathrm{mI} \\
(\mathrm{n}=31)\end{array}$ & $\mathrm{L}$ \\
\hline Age, years & & & & 0.624 \\
\hline$\leq 50$ & 49 & 37 & 12 & \\
\hline$>50$ & 91 & 72 & 19 & \\
\hline Sex & & & & $0.285^{\dagger}$ \\
\hline Male & 116 & 88 & 28 & \\
\hline Female & 24 & 21 & 3 & \\
\hline $\mathrm{HBsAg}$ & & & & $0.766^{\dagger}$ \\
\hline Negative & 13 & 10 & 3 & \\
\hline Positive & 127 & 99 & 28 & \\
\hline Child-Pugh score & & & & $0.607^{\dagger}$ \\
\hline A & 133 & 103 & 30 & \\
\hline $\mathrm{B}$ & 7 & 6 & 1 & \\
\hline Liver cirrhosis & & & & $0.130^{\dagger}$ \\
\hline No & 28 & 25 & 3 & \\
\hline Yes & 112 & 84 & 28 & \\
\hline ALT, U/L & & & & 0.223 \\
\hline$\leq 75$ & 94 & 76 & 18 & \\
\hline$>75$ & 46 & 33 & 13 & \\
\hline AFP, ng/mL & & & & 0.485 \\
\hline$\leq 400$ & 106 & 84 & 22 & \\
\hline$>400$ & 34 & 25 & 9 & \\
\hline No. of tumors & & & & 0.234 \\
\hline Single & 118 & 94 & 24 & \\
\hline Multiple & 22 & 15 & 7 & \\
\hline Tumor size, $\mathrm{cm}$ & & & & 0.442 \\
\hline$\leq 5$ & 119 & 94 & 25 & \\
\hline$>5$ & 21 & 15 & 6 & \\
\hline Tumor encapsulation & & & & 0.71 \\
\hline Complete & 49 & 39 & 10 & \\
\hline None & 91 & 70 & 21 & \\
\hline Satellite lesion & & & & 0.684 \\
\hline No & 131 & 101 & 30 & \\
\hline Yes & 9 & 8 & 1 & \\
\hline Vascular invasion & & & & 0.492 \\
\hline No & 93 & 74 & 19 & \\
\hline Yes & 47 & 35 & 12 & \\
\hline Edmondson stage & & & & 0.85 \\
\hline I-II & 102 & 79 & 23 & \\
\hline III-IV & 38 & 30 & 8 & \\
\hline Recurrence & & & & $<0.001$ \\
\hline No & 101 & 87 & 14 & \\
\hline Yes & 39 & 22 & 17 & \\
\hline
\end{tabular}

*ALT, alanine aminotransferase; HBsAg, hepatitis B surface antigen; 'Fisher's exact test

of $18.78 \pm 0.69$ months (95\% CI 17.42-20.14 months). The levels of PIVKA-II were significantly higher in patients with early recurrence than in those without early recurrence (median $28.25 \mathrm{mAU} / \mathrm{mL}$, interquartile range 55-314.25; mean 819.8, SD $2959.69 \mathrm{mAU} / \mathrm{mL} ; p=0.039$; (Figure 1). As expected, the median concentration of AFP in serum did not differ between patients with and without early recurrence ( $p=0.188$ ). (Figure 2 ) shows the ROC curves for all patients with and without early recurrence. The area under the ROC curves (AUROC) for PIVKA-II was 0.653 . The cutoff value was $373.5 \mathrm{mAU} / \mathrm{mL}$. One hundred and nine patients had PIVKA-II level $<373.5 \mathrm{mAU} / \mathrm{mL}$, and $31 \mathrm{had} \geq 373.5 \mathrm{mAU} / \mathrm{mL}$. Levels of PIVKA-II were significantly higher in patients with recurrence than in those without recurrence. Recurrence was observed in 17 of 31 patients with preoperative PIVKA-II level $\geq 373.5$ $\mathrm{mAU} / \mathrm{mL}$, whereas only 22 of 109 patients with $<373.5$ $\mathrm{mAU} / \mathrm{mL}$ had recurrence $(p<0.001$, Table 2$)$.
A
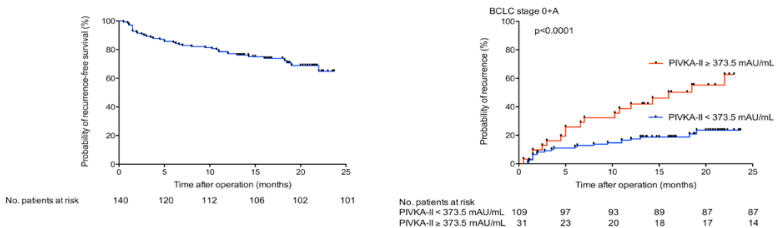

Figure 3. PIVKA-II levels Correlated Significantly with Early Recurrence in BCLC Stage 0-A Patients. A) Overall recurrence-free survival curve of 140 BCLC stage 0 -A patients who underwent curative hepatic resection B) Kaplan-Meier analysis for time to recurrence in HCC patients with PIVKA-II $\geq 373.5 \mathrm{mAU} / \mathrm{mL}$ or $<373.5 \mathrm{~m}$ AU/ $\mathrm{mL}$, preoperatively

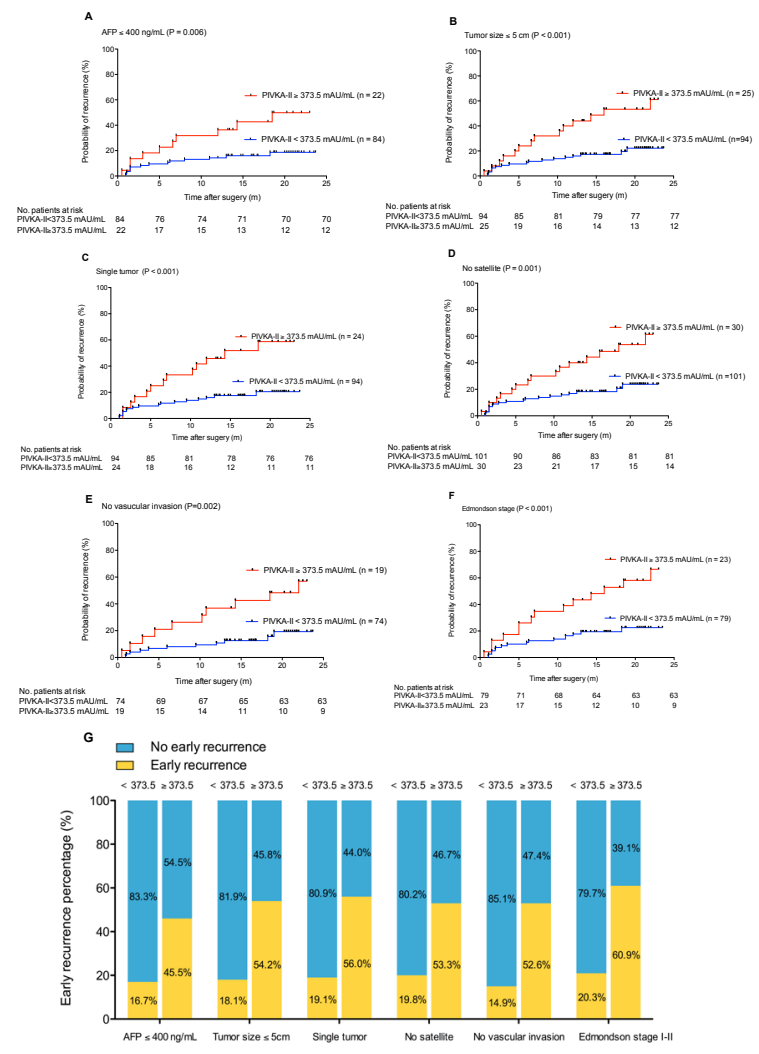

Figure 4. Kaplan-Meier Analysis of PIVKA-II Levels in Subgroups of BCLC 0-A HCC Patients. The prognostic value of PIVKA-II levels was significant in A) AFP $\leq 400 \mathrm{ng} / \mathrm{mL}$ and B-F) clinical low-recurrence-risk subgroups, including B) tumor size $\leq 5 \mathrm{~cm}, C$ ) single tumor, D) no satellite lesions, E) no vascular invasion, and F) Edmondson stage I/II. G) Recurrent rates of patients with PIVKA-II levels $<373.5 \mathrm{mAU} / \mathrm{mL}$ versus $\geq 373.5 \mathrm{mAU} / \mathrm{mL}$ in the subgroups for AFP $\leq 400 \mathrm{ng} / \mathrm{mL}$, tumor size $\leq 5 \mathrm{~cm}$, single tumor, no satellite lesion, no vascular invasion, and Edmondson stage I/II

\section{Survival}

The 1- and 2-year disease-free survival rates were 77.1 and $72.1 \%$, respectively (Figure $3 \mathrm{~A}$ ). Patients with PIVKA-II level $>373.5 \mathrm{mAU} / \mathrm{mL}$ had significantly shorter TTR and higher recurrence rates $(54.8 \%$ vs $20.2 \%)$ than those with PIVKA-II level $<373.5 \mathrm{mAU} / \mathrm{mL}(p<0.0001)$ (Figure 3B). Independent predictors of tumor recurrence by univariate analysis were associated with PIVKA-II 
Table 2. Univariate and Multivariate Cox Proportional Regression Analysis of Factors Associated with Recurrence

\begin{tabular}{|c|c|c|c|c|}
\hline & \multicolumn{2}{|c|}{ Univariate analysis } & \multicolumn{2}{|c|}{ Multivariate analysis } \\
\hline & $\operatorname{HR}(95 \% \mathrm{CI})$ & $p$ & $\mathrm{HR}(95 \% \mathrm{CI})$ & $p$ \\
\hline Age, $>50 v s \leq 50$ years & $0.538(0.286-1.013)$ & 0.055 & NA & NA \\
\hline Sex, male $v s$ female & $1.323(0.517-3.384)$ & 0.559 & NA & NA \\
\hline HBsAg, positive $v s$ negative & $1.388(0.332-5.799)$ & 0.653 & NA & NA \\
\hline Liver cirrhosis, yes $v s$ no & $3.271(1.007-10.627)$ & 0.049 & NA & NA \\
\hline Child-Pugh score, B vs A & $2.007(0.616-6.543)$ & 0.248 & NA & NA \\
\hline $\mathrm{ALT},>75 \mathrm{U} / \mathrm{L} v s \leq 75 \mathrm{U} / \mathrm{L}$ & $1.272(0.667-2.427)$ & 0.465 & NA & NA \\
\hline $\mathrm{AFP},>400 \mathrm{ng} / \mathrm{mL} v s \leq 400 \mathrm{ng} / \mathrm{mL}$ & $2.123(1.112-4.053)$ & 0.023 & NA & NA \\
\hline No. of tumors, multiple $v s$ single & $1.507(0.711-3.192)$ & 0.285 & NA & NA \\
\hline Tumor size, $>5 \mathrm{~cm} v s \leq 5 \mathrm{~cm}$ & $1.574(0.686-3.608)$ & 0.284 & NA & NA \\
\hline Tumor encapsulation, none $v s$ complete & $1.233(0.624-2.437)$ & 0.546 & NA & NA \\
\hline Satellite lesion, yes vs no & $1.155(0.355-3.754)$ & 0.811 & NA & NA \\
\hline Vascular invasion, yes $v s$ no & $2.093(1.109-3.951)$ & 0.023 & $2.02(1.524-3.836)$ & 0.032 \\
\hline Edmondson stage, III/IV vs I/II & $0.814(0.386-1.714)$ & 0.588 & NA & NA \\
\hline Preoperative PIVKA-II, $>373.5 v s \leq 373.5 \mathrm{AU} / \mathrm{mL}$ & $2.965(1.572-5.595)$ & 0.001 & $2.877(1.524-5.429)$ & 0.001 \\
\hline
\end{tabular}
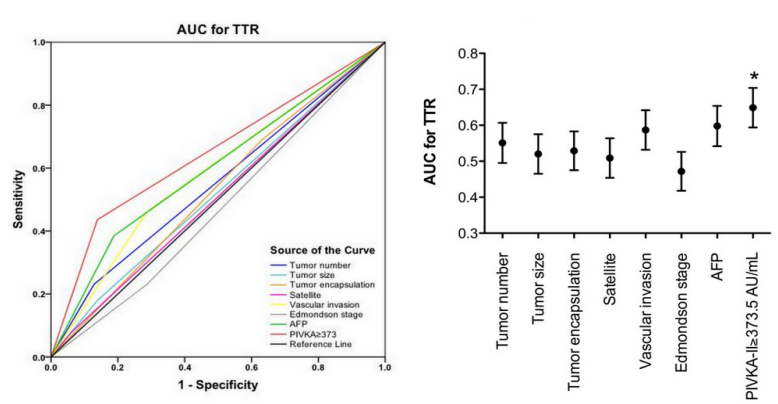

Figure 5. Predictive Ability of PIVKA-II $\geq 373.5 \mathrm{mAU} /$ mL was Compared with Other Clinical Parameters by ROC Curves in 140 BCLC Stage 0-A HCC Patients. The AUC with $95 \% \mathrm{CI}$ for time to recurrence are also shown. ${ }^{*} p<0.05$ versus PIVKA-II $\geq 373.5 \mathrm{mAU} / \mathrm{mL}$

$373.5 \mathrm{mAU} / \mathrm{mL}$, elevated alanine aminotransferase, AFP, tumor size, tumor encapsulation, satellite lesion, vascular invasion, Edmondson stage, and level of PIVKA-II (Table 2). Level of AFP, liver cirrhosis, vascular invasion, and level of PIVKA-II were unfavorable prognostic variables for recurrence $(p<0.05)$ (Table 2$)$. In multivariate analysis, PIVKA-II $373.5 \mathrm{mAU} / \mathrm{mL}$ and vascular invasion (HR, 2.020; 95\% CI 1.524-3.836; $p=0.032$ ) were independent prognostic factor for TTR, and PIVKA-II $373.5 \mathrm{mAU} /$ $\mathrm{mL}$ was the strongest independent prognostic factor (HR, 2.877; 95\% CI 1.524-5.429; $p=0.001$, Table 2).

The prognostic significance of preoperative PIVKAII $373.5 \mathrm{mAU} / \mathrm{mL}$ within clinical subgroups was further investigated. In patients with AFP $\leq 400 \mathrm{ng} / \mathrm{mL}$, patients with PIVKA-II level $\geq 373.5 \mathrm{mAU} / \mathrm{mL}$ had higher recurrence rates $(45.5 \%$ vs $16.7 \%)$ and shorter TTR than those with PIVKA-II level $<373.5 \mathrm{mAU} / \mathrm{mL}$ ( $p=0.006)$ (Figure 4A). Patients with preoperative PIVKA-II level $\geq 373.5 \mathrm{mAU} / \mathrm{mL}$ showed a relatively higher risk of developing postoperative recurrence than those with PIVKA-II level $<373.5 \mathrm{mAU} / \mathrm{mL}$ in low-recurrence-risk subgroups, including tumor size $\leq 5 \mathrm{~cm}(54.2 \%$ vs $18.1 \%$; $p<0.001)$, single tumor $(56.0 \%$ vs $19.1 \%$; $p<0.001)$, absence of satellite lesions $(53.3 \%$ vs $19.8 \% ; p<0.001)$, absence of vascular invasion ( $52.6 \%$ vs $14.9 \%$; $p=0.002)$, and Edmondson stage I/II (60.9\% vs 20.3\%; $p<0.001)$, (Figure 4B-G).
ROC analysis showed that the AUC for the level of PIVKA-II was 0.649 , with a sensitivity of $43.6 \%$ and specificity of $86.1 \%$ ( $p=0.006 ; 95 \%$ CI $0.540-0.757)$. Compared with other clinical indices, the level of PIVKAII prior to resection was the strongest factor for predicting early recurrence in HCC (AUCs with 95\% CI for TTR; $p<0.05$ vs PIVKA-II $373.5 \mathrm{mAU} / \mathrm{mL}$ ) (Figure 5). The predictive power of the simplified model was higher than the single factors of level of PIVKA-II and vascular invasion.

\section{Discussion}

The most effective therapeutic options for HCC offering a favorable prognosis are hepatectomy and liver transplantation. However, even such curative surgery does not guarantee full recovery, and this failure is owing in large part to the high incidence of recurrence (50$70 \%$ at 5 years) (Cha et al., 2003). The most significant reason for the unsatisfactory therapeutic outcome is residual micrometastases formed prior to resection, or dissemination of tumor cells during surgical manipulation (Shah et al., 2007). Unfortunately, routine diagnostic approaches are thus far unable to identify the HCC patient subpopulation at high risk of developing micrometastases preoperatively (Shan et al., 2006). Increasing attention has been poured into developing markers for predicting HCC recurrence risk. For instance, The fibroblast growth factorinducible 14 (Fn14) expression, closely associated with AFP, can be believed to indicate poor surgical outcome when it is detected overexpressed (Li et al., 2013). The low counts of $\gamma \delta$ T cells in peritumoral liver tissue suggest a higher incidence of recurrence in HCC and are good to postoperative recurrence, especially in patients with early-stage HCC (Cai et al., 2014).

For patients with relevant portal hypertension (5-year survival: $50 \%$, BCLC stage A2) or both adverse prognostic factors (5-year survival: 25\%, BCLC stage A3), BCLC is the sole system that links staging to treatment indication. Very early HCC stage (BCLC 0 ) is defined as patients with well-preserved liver function diagnosed with the carcinoma in situ, which mostly involves a single HCC lesion $<2 \mathrm{~cm}$. The best candidates for resection are patients 
with single asymptomatic $\mathrm{HCC}$ with preserved liver function (BCLC 0-A), which may achieve $70 \%$ survival rates after treatment (Lovet et al., 1999).

PIVKA-II is an aberrant form of prothrombin produced by HCC cells. Recently, The effects of PIVKA-II on growth and migration of human vascular endothelial cells (HUVECs) was demonstrated (Wang et al., 2009a). They found that PIVKA-II significantly stimulated the proliferation of HUVECs (ECV304 cells) in a dose- and time-dependent manner and expression of epidermal growth factor receptor, vascular endothelial growth factor, and matrix metalloproteinase-2. Anatomical resection appeared to have a beneficial effect on recurrence-free survival after hepatectomy for HCC, and PIVKA-II measurement was effective in predicting $\mathrm{HCC}$ recurrence and had the advantage that it can be assessed before surgery (Yamamoto et al., 2010). It was suggested that PIVKA-II is a useful tumor marker for HCC, complementary to AFP (Kim et al., 2007). Serial measurements of both markers after resection might be helpful for early diagnosis of tumor recurrence. Furthermore, combination of PIVKAII and AFP improves initial diagnosis of HCC, and the sensitivity of these markers is greatest at the time of HCC identification and noticeably less so at earlier time points (Mittal et al., 2012). Our study suggests that PIVKA-II is useful for early diagnosis of BCLC 0-A HCC recurrence. However, our study showed that AFP was only a risk factor (HR, 2.123; 95\% CI 1.112-4.053; $p=0.023$ ), and not an independent risk factor of BCLC 0-A HCC early recurrence. PIVKA-II $373.5 \mathrm{mAU} / \mathrm{mL}$ was the strongest independent prognostic factor $(\mathrm{HR}, 2.877$; 95\% CI 1.5245.429; $p=0.001$, Table 2). Thus, the preoperative detection of serum PIVKA-II level might serve as a novel indicator reflecting early recurrence of BCLC 0 -A HCC. In clinical practice, it is challenging to predict tumor relapse in the lowest-recurrence-risk HCC subgroups (Tung-Ping Poon et al., 2000; Shah et al., 2006). The present study is the first to show that preoperative serum PIVKA-II level retains its prognostic value in subgroups for which conventional clinicopathological variables offer limited prediction of tumor recurrence. So far, AFP level is the most extensively used diagnostic biomarker and tumor recurrence indicator of HCC (Chan et al., 2009). Clinical data demonstrated that low serum AFP concentration (e.g., $<400 \mathrm{ng} / \mathrm{mL}$ ) was associated with better clinical outcome. Nevertheless, it is difficult to monitor recurrence in the $30-40 \%$ of $\mathrm{HCC}$ patients with low AFP levels (Shan et al., 2006; Wang et al., 2009b). It was demonstrated that HCC patients with low values of both AFP and PIVKA-II had more favorable clinical characteristics and showed better prognosis than those with elevated levels of AFP or PIVKA-II (Kang et al., 2012). Combination of AFP and PIVKA-II response has predictive power for PFS and overall survival comparable to radiological criteria and better than AFP response alone (Park et al., 2013). Here, we showed that determination of preoperative serum PIVKA-II level is a promising and feasible marker for prediction of recurrence in patients with low AFP concentration $(p=0.006)$. Large cohort studies should be undertaken to validate the prognostic significance in this specific HCC patient subpopulation. Furthermore, in clinicopathologically lower-risk patients (tumor size $\leq 5 \mathrm{~cm}$, single tumor, no satellite lesion, no vascular invasion, Edmondson stage I/II, and BCLC stage 0 -A), those with serum PIVKA-II $<373.5 \mathrm{mAU} / \mathrm{mL}$ postoperatively showed lower recurrence risk than those with PIVKA-II $\geq 373.5 \mathrm{mAU} / \mathrm{mL}(p \leq 0.002)$. We propose that determination of serum PIVKA-II level may provide a powerful test enabling accurate and early decision making to tailor the most effective therapy according to characteristics of individual tumors.

The limitations of this study were its relatively small cohort size, short follow-up time, and data from a single study center. Most patients with HCC in China have hepatitis $\mathrm{B}$ and the predictive model needs validation in patients with HCC from those areas. Additionally, the findings need a larger population prospective study to validate their usefulness.

To the best of our knowledge, this is the first study to identify the levels of PIVKA-II in BCLC 0-A patients. BCLC 0-A HCC is generally associated with good prognosis. Preoperative level of PIVKA-II > $373.5 \mathrm{mAU} /$ $\mathrm{mL}$ is an independent prognostic indicator for recurrence in BCLC 0-A HCC patients undergoing curative resection. In patients with low AFP level, PIVKA-II will improve the finding of early recurrence of HCC.

\section{Acknowledgements}

This study was supported by grants from the State Key Laboratory of Clinical College Construction Project (/), the National Science \& Technology Pillar Program during the 12th Five-year Plan Period (2012BAI37B01), and the Research Fund of Tumorigenesis and Invasion Principle Key Laboratory of Ministry of Education (/).

\section{References}

Bertino G,Ardiri A, Malaguarnera M, et al (2012). Hepatocellualar carcinoma serum markers. Semin Oncol, 39, 410-33.

Bruix J, Sherman M (2011). Management of hepatocellular carcinoma: an update. Hepatology, 53, 1020-2.

Cai XY, Wang JX, Yi Y, et al (2014). Low counts of $\gamma \delta$ T cells in peritumoral liver tissue are related to more frequent recurrence in patients with hepatocellular carcinoma after curative resection. Asian Pac J Cancer Prev, 15, 775-80.

Cha C, Fong Y, Jarnagin WR, et al (2003). Predictors and patterns of recurrence after resection of hepatocellular carcinoma. $J$ Am Coll Surg, 197, 753-8.

Chan SL, Mo FK, Johnson PJ, et al (2009). New utility of an old marker: serial-fetoprotein measurement in predicting radiologic response and survival of patients with hepatocellular carcinoma undergoing systemic chemotherapy. J Clin Oncol, 27, 446-52.

Gupta SP, Mittal A, Sathian B, et al (2013). Elevated serum insulin is an independent risk factor for hepatocellular carcinoma: a case control study from Nepal. Asian Pac J Cancer Prev, 14, 7331-3.

Kang SH, Kim do Y, Jeon SM, et al (2012). Clinical characteristics and prognosis of hepatocellular carcinoma with different sets of serum AFP and PIVKA-II levels. Eur J Gastroenterol Hepatol, 24, 849-56.

Kim do Y, Paik YH, Ahn SH, et al (2007). PIVKA-II is a useful tumor marker for recurrent hepatocellular carcinoma after surgical resection. Oncology, 72, 52-7. 
Kim JM, Hyuck C, Kwon D, et al (2013). Protein induced by vitamin $\mathrm{K}$ antagonist-II (PIVKA-II) is a reliable prognostic factor in small hepatocellular carcinoma. World J Surg, 37, 1371-8.

Li N, Hu WJ, Shi J, et al (2013). Roles of fibroblast growth factor-inducible 14 in hepatocellular carcinoma. Asian Pac $J$ Cancer Prev, 14, 3509-14.

Lovet JM, Brú C, Bruix J (1999). Prognosis of hepatocellular carcinoma: the BCLC staging classification. Semin Liver Dis, 19, 329-38.

Matsubara M, Shiraha H, Kataoka J, et al (2012). Des- $\gamma$-carboxyl prothrombin is associated with tumor angiogenesis in hepatocellular carcinoma. J Gastroenterol Hepatol, 27, 1602-8.

Mittal A, Gupta SP, Sathian B, et al (2012). Des-gammacarboxyprothrombin for early identification and prognosis of hepatocellular carcinoma: a case control study from western Nepal. Asian Pac J Cancer Prev, 13, 5773-5.

Park H, Park JY (2013). Clinical significance of AFP and PIVKA-II responses for monitoring treatment outcomes and predicting prognosis in patients with hepatocellular carcinoma. Biomed Res Int, 310427.

Santi V, Trevisani F, Gramenzi A, et al (2010). Semiannual surveillance is superior to annual surveillance for the detection of early hepatocellular carcinoma and patient survival. J Hepatol, 53, 291-7.

Shah SA, Greig PD, Gallinger S, et al (2006). Factors associated with early recurrence after resection for hepatocellular carcinoma and outcomes. J Am Coll Surg, 202, 275-83.

Shah SA, Cleary SP, Wei AC, et al (2007). Recurrence after liver resection for hepatocellular carcinoma: risk factors, treatment, and outcomes. Surgery, 141, 330-9.

Shi L, Wu LL, Yang JR, et al (2014). Serum peroxiredoxin3 is a useful biomarker for early diagnosis and assessemnt of prognosis of hepatocellular carcinoma in Chinese patients. Asian Pac J Cancer Prev, 15, 2979-86.

Siegel R, Naishadham D, Jemal A (2012). Cancer statistics, 2012. CA Cancer J Clin, 62, 10-29.

Takayama T, Makuuchi M, Kojiro M, et al (2008). Early hepatocellular carcinoma: pathology, imaging, and therapy. Ann Surg Oncol, 15, 972-8.

Tung-Ping Poon R, Fan ST, Wong J (2000). Risk factors, prevention, and management of postoperative recurrence after resection of hepatocellular carcinoma. Ann Surg, 232, 10-24.

Wang SB, Cheng YN, Cui SX, et al (2009a). Des-gammacarboxy prothrombin stimulates human vascular endothelial cell growth and migration. Clin Exp Metastasis, 26, 469-77.

Wang CC, Iyer SG, Low JK, et al (2009b). Perioperative factors affecting long-term outcomes of 473 consecutive patients undergoing hepatectomy for hepatocellular carcinoma. Ann Surg Oncol, 16, 1832-42.

Wang NY, Wang C, Li W, et al (2014). Prognostic value of serum AFP, AFP-L3, and GP73 in monitoring short-term treatment response and recurrence of hepatocellular carcinoma after radiofrequency ablation. Asian Pac J Cancer Prev, 15, 1539-44.

Yamamoto K, Imamura H, Matsuyama Y, et al (2010). AFP, AFP-L3, DCP, and GP73 as markers for monitoring treatment response and recurrence and as surrogate markers of clinicopathological variables of HCC. J Gastroenterol, 45, 1272-82. 